\title{
Performance of a SIMO-CDSK System over Rayleigh Fading Channels
}

\author{
Jun-Yi Duan, ${ }^{1,2}$ Guo-Ping Jiang, ${ }^{3}$ and Hua Yang ${ }^{3}$ \\ ${ }^{1}$ School of Communication and Information Engineering, Nanjing University of Posts and Telecommunications, Nanjing 210003, China \\ ${ }^{2}$ Nanjing Institute of Railway Technology, Nanjing 210031, China \\ ${ }^{3}$ School of Automation, Nanjing University of Posts and Telecommunications, Nanjing 210003, China
}

Correspondence should be addressed to Jun-Yi Duan; duanjunyi922@126.com

Received 6 September 2013; Revised 24 October 2013; Accepted 3 November 2013

Academic Editor: Hai Yu

Copyright (C) 2013 Jun-Yi Duan et al. This is an open access article distributed under the Creative Commons Attribution License, which permits unrestricted use, distribution, and reproduction in any medium, provided the original work is properly cited.

\begin{abstract}
This paper proposes a single-input multiple-output (SIMO) architecture for correlation delay shift keying (CDSK) modulation technique, and the bit error rate (BER) formula is derived under the assumption of the proposed system over Rayleigh fading channels. The new system employs multiple antennas at the receiver end to form a SIMO structure so as to obtain a diversity gain. Theoretical analysis and simulations show that, at a higher signal-to-noise ratio (SNR), the proposed SIMO-CDSK architecture has an outstanding bit error rate (BER) performance in contrast to the conventional single-input single-output (SISO) CDSK and GCDSK communication system; for the given SNR, the diversity gain of the proposed system will be improved with the number of receiver antennas increasing; for different SNRs, the best performance of the proposed system can be obtained by selecting the reasonable spreading factor; because the performance of SIMO-CDSK system is independent of the time delay, the proposed system has better security than GCDSK system.
\end{abstract}

\section{Introduction}

Chaotic maps/systems, with the favorable properties of being aperiodic, deterministic, noise-like, and wideband, have been widely employed in various modulation applications. During the last two decades or so, lots of digital communication schemes using chaos have been suggested and studied, which utilize either coherent or noncoherent detections for information extraction [1]. Because chaotic synchronization in coherent detection performs poorly, demodulation in chaosbased communication schemes with coherent receivers using this synchronization technique becomes a real challenge in noisy environments. Therefore, noncoherent schemes seem more feasible and appealing in practical environments. In 1996, Kolumbán et al. proposed the noncoherent differential chaos shift keying (DCSK) which is the most suitable scheme in wireless communications due to its good noise performance $[2,3]$. However, the prices that it has to pay are low attainable data rate and weakened information security. In 2000, Sushchik et al. proposed the noncoherent correlation delay shift keying (CDSK) whose bandwidth utilization ratio and information security are much better than DCSK, but it is inferior in its bit error rate (BER) performances [4]. For this reason, the generalized CDSK (GCDSK) has been introduced so as to offer solution with improved bandwidth efficiency and BER but leading to a more complicated system design in hardware [5]. Furthermore, frequency-modulated CDSK (FM-CDSK) [6] and CDSK with Henon chaotic map [7] have been researched in recent years; these schemes further improve the security and practicality of CDSK.

The use of multiple antennas at all terminals or only the receiver end, which are called multiple input multiple output (MIMO) or single-input multiple-output (SIMO) techniques, demonstrates an effective enhancement in link reliability and bandwidth efficiency [8]. As a potential application, applying MIMO technique to chaos-based communication system has been considered. Due to the advantage of low BER, SIMODCSK system and MIMO-DCSK system have been carefully studied aiming to improve the performance of DCSK system over fading channels $[9,10]$. In addition, a SIMO FM-DCSK 


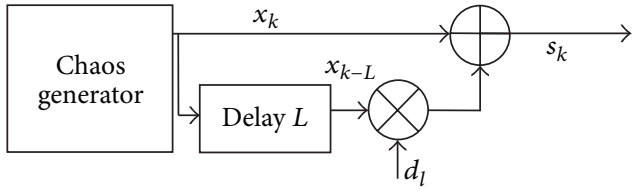

(a)

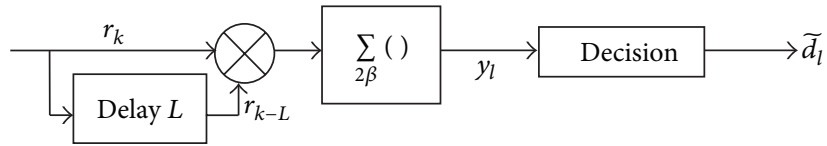

(b)

FIGURE 1: CDSK modulation: (a) transmitter and (b) receiver.

UWB system has been proposed to further improve the performance in such environment [11].

However, little research has been conducted focusing on the performance improvement of CDSK modulation combined with multiantennas technique. To address this problem, an advanced CDSK system, single-input multipleoutput CDSK (SIMO-CDSK) system, is proposed in present paper. Here, multiple antennas are allocated to the receiver end and provide several independent copies of signals from the transmitter. The BER formula is also derived under the assumption of the proposed system over Rayleigh fading channels. Theoretical analysis and simulations show that, at a higher signal-to-noise ratio (SNR), the proposed SIMOCDSK architecture has an outstanding bit error rate performance in contrast to the conventional single-input singleoutput CDSK (SISO-CDSK) system and GCDSK system; for the given SNR, the diversity gain of the proposed system will be improved with the number of receiver antennas increasing; for different SNRs, the best performance of the proposed system can be obtained by selecting the reasonable spreading factor; because the performance of SIMO-CDSK system is independent of the time delay, the proposed system has better security than GCDSK system.

The remainder of this paper is organized as follows. In Section 2, the principle of CDSK modulation is briefly reviewed, while SIMO-CDSK system is described. In Section 3, performance analysis on SIMO-CDSK system over Rayleigh fading channels is carried out, with a close form BER expression derived. Simulation results are shown in Section 4 and conclusions are given in Section 5.

\section{System Model}

In this section, a single-input multiple-output CDSK system is introduced based on the principle of CDSK modulation. In the rest of this paper, it is assumed that all the independent channels satisfy the same Rayleigh fading channel condition and the same Gaussian-distributed random noise with the power spectral density of $N_{0}$. Also, equal-gain combiner (EGC) is adopted at the receivers in this system because it not only can improve the error performance but also can be easily implemented.

2.1. Modulation of CDSK. CDSK was proposed by Sushchik et al. to transmit information and to increase the efficiency of bandwidth [4].

As seen in Figure 1, in this scheme, the chaotic signal is employed as the carrier for transmission. When the chaotic signal is generated by a discrete time chaotic circuit, the transmitted signal is the sum of a chaotic sequence $x_{k}$ and of the delayed chaotic sequence $x_{k-L}$ multiplied by the $l$ th transmitted information signal $d_{l}= \pm 1$. During the $l$ th bit period, as seen from Figure 1(a), the output of the transmitter $s_{k}$ is

$$
s_{k}=x_{k}+d_{l} x_{k-L},
$$

where $L$ is the time delay. Assume that the transmitted signal will suffer from Rayleigh fading and the influence of Gaussian-distributed random noise. According to Figure 1(b), the received signal corresponding to the $l$ th transmitted information signal $d_{l}$ is given by

$$
r_{k}=h_{k} s_{k}+\xi_{k}
$$

where $h_{k}$ is the independent and Rayleigh distribution random variable and $\xi_{k}$ is a sequence of independent and identically distributed zero-mean Gaussian noise variables. During the period of the $l$ th transmitted information signal, the correlator output of CDSK $y_{l}$ is given by the sum

$$
y_{l}=\sum_{k=2(l-1) \beta+1}^{2 l \beta} r_{k} r_{k-L},
$$

where $2 \beta$ is the spreading factor. The estimated information is decided as " +1 " or " -1 " depending on the following rules:

$$
\widetilde{d}= \begin{cases}+1, & y_{l} \geq 0, \\ -1, & y_{l}<0 .\end{cases}
$$

2.2. SIMO-CDSK System Model. For digital chaotic communication, CDSK has advantages in bandwidth utilization ratio and information security. However, the inferiority in bit error rate limits its application and popularization. The use of multiple antennas at the receiver end, which is called singleinput multiple-output (SIMO) technique, demonstrates a significant improvement in link reliability and bandwidth efficiency. Motivated by the virtue of CDSK and SIMO technology, SIMO-CDSK system is proposed to enhance the performance of the conventional SISO-CDSK system. Figure 2 shows the block diagram of the SIMO-CDSK system, where (a) is the transmitter and (b) is the receiver.

In this proposed system, we consider that multiple antennas are allocated to the receiver end. In order to generate the transmitted signal, the modulator is shown in Figure 2(a), which appears to be the identical version of CDSK modulation. The channels constructed by the transmitter-receiver 


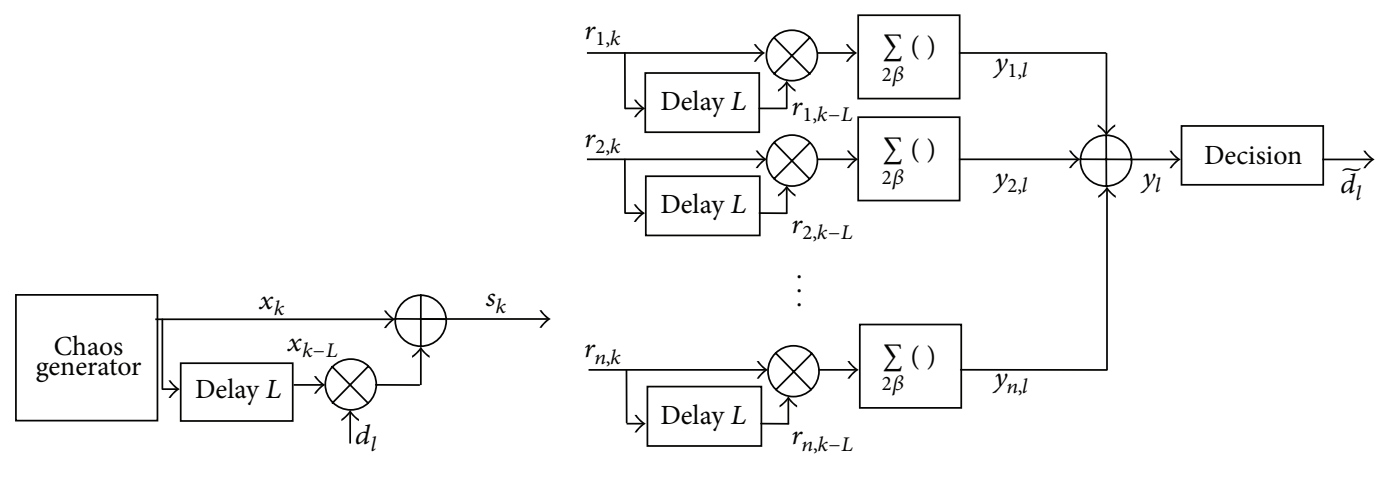

(a)

(b)

FIGURE 2: SIMO-CDSK system: (a) transmitter and (b) receiver.

antenna pairs are independent, meaning that the channel state remains constant during each transmission period.

In SIMO-CDSK system, we assume that all channels satisfy the same Rayleigh fading channel condition and the additive white Gaussian noise which is independent from channel. Consequently, according to Figure 2(b), the received signals at the $i$ th receiver antenna which corresponding to the $l$ th transmitted information signal $d_{l}$ can be expressed as follow:

$$
r_{i, k}=h_{i, k} s_{k}+\xi_{i, k} \text {, }
$$

where $h_{i, k}$ and $\xi_{i, k}$ are parameters of the channel which are between the transmitter antenna and the $i$ th receiver antenna, $h_{i, k}$ is the independent and Rayleigh distributed random variable, and $\xi_{i, k}$ is a sequence of independent and identically distributed Gaussian noise. With expressions shown in (5), under the Rayleigh fading channel model, the $i$ th correlator output of SIMO-CDSK system $y_{i, l}$ is

$$
y_{i, l}=\sum_{k=2(l-1) \beta+1}^{2 l \beta}\left(h_{i, k} s_{k}+\xi_{i, k}\right)\left(h_{i, k-L} s_{k-L}+\xi_{i, k-L}\right) \text {, }
$$

where $2 \beta$ is the spreading factor. To obtain the performance gain, as seen from Figure 2(b), the equal-gain combiner (EGC) is adopted at the receiver in this proposed system. Therefore, by equally combining the correlator outputs, the sum of correlator outputs $y_{l}$ is used to make the decision for $d_{l}$. Under the Rayleigh fading channel model, the expression of $y_{l}$ can be derived as

$$
y_{l}=\sum_{i=1}^{n} y_{i, l}
$$

where $n$ is the number of receiver antennas. As shown in (4), the estimated information is decided as " +1 " or " -1 " depending on $y_{l}$.

\section{Performance Analysis}

In this section, the BER expression of SIMO-CDSK system is derived over Rayleigh fading channels. To simplify the calculation of the bit error rate, in the following analysis, we assume that the time delay $L$ is greater than the spreading factor $2 \beta$.

With the expression of $y_{l}$, correlator output in (7) can be rewritten as

$$
\begin{aligned}
y_{l}= & \sum_{i=1}^{n}\left[\sum_{k=2(l-1) \beta+1}^{2 l \beta}\left(h_{i, k} h_{i, k-L} s_{k} s_{k-L}\right)\right] \\
& +\sum_{i=1}^{n}\left[\sum_{k=2(l-1) \beta+1}^{2 l \beta}\left(h_{i, k} s_{i, k} \xi_{i, k-L}+h_{i, k-L} s_{i, k-L} \xi_{i, k}\right)\right] \\
& +\sum_{i=1}^{n}\left[\sum_{k=2(l-1) \beta+1}^{2 l \beta}\left(\xi_{i, k} \xi_{i, k-L}\right)\right]=A+B+C,
\end{aligned}
$$

where

$$
\begin{gathered}
A=\sum_{i=1}^{n}\left[\sum_{k=2(l-1) \beta+1}^{2 l \beta}\left(h_{i, k} h_{i, k-L} s_{k} s_{k-L}\right)\right], \\
B=\sum_{i=1}^{n}\left[\sum_{k=2(l-1) \beta+1}^{2 l \beta}\left(h_{i, k} s_{i, k} \xi_{i, k-L}+h_{i, k-L} s_{i, k-L} \xi_{i, k}\right)\right], \\
C=\sum_{i=1}^{n}\left[\sum_{k=2(l-1) \beta+1}^{2 l \beta}\left(\xi_{i, k} \xi_{i, k-L}\right)\right] .
\end{gathered}
$$

As depicted in (8), $A$ means the signal component which is used to make the decision for $d_{l}$, and $B$ means the interference component which is produced by the correlation between the transmitted signal and white Gaussian noise. $C$ means the interference component which is merely produced by the white Gaussian noise.

In this work, we assume that parallel channels are slow fading, the period of $h_{i, k}$ stays constant which is much greater than the time delay. Thus, we can then represent the fading phenomenon using a random variable whose value is fixed; that is,

$$
h_{i, k}=h_{i, k-L}=h_{i} \text {, }
$$


where $i$ means the $i$ th channel. For large $2 \beta$ and a given chaotic map (e.g., chebyshev map [12]), we have

$$
\sum_{k=2(l-1) \beta+1}^{2 l \beta} x_{k} x_{k-L}=0 .
$$

Assuming " +1 " is transmitted (i.e., $d_{l}=+1$ ), the following statistics are easily obtained:

$$
\begin{array}{r}
E\left[y_{l} \mid H, d_{l}=+1\right]=\sum_{i=1}^{n} 2 \beta h_{i}^{2} E\left[x_{k}^{2}\right], \\
\operatorname{Var}\left[y_{l} \mid H, d_{l}=+1\right] \\
=2 \beta\left[\sum_{i=1}^{n} h_{i}^{4}\left(3\left(E\left[x_{k}^{2}\right]\right)^{2}+\operatorname{Var}\left[x_{k}^{2}\right]\right)\right. \\
\left.+2 \sum_{i=1}^{n} h_{i}^{2} E\left[x_{k}^{2}\right] N_{0}+n\left(\frac{N_{0}}{2}\right)^{2}\right],
\end{array}
$$

where $H$ means the set of channel fading parameters and $E[\cdot]$ and $\operatorname{Var}[\cdot]$ represent the expectation and variance operators, respectively. Since $\operatorname{Var}\left[y_{l} \mid H, d_{l}=+1\right]$ is mainly affected by the white Gaussian noise when $2 \beta$ is relatively small, the value which is calculated by $\left(3\left(E\left[x_{k}^{2}\right]\right)^{2}+\operatorname{Var}\left[x_{k}\right]\right)$ is relatively small; (12) may be approximated as

$$
\begin{gathered}
\operatorname{Var}\left[y_{l} \mid H, d_{l}=+1\right] \\
\approx 2 \beta\left[\left(\sum_{i=1}^{n} h_{i}^{2}\right)^{2}\left(3\left(E\left[x_{k}^{2}\right]\right)^{2}+\operatorname{Var}\left[x_{k}^{2}\right]\right)\right. \\
\left.+2 \sum_{i=1}^{n} h_{i}^{2} E\left[x_{k}^{2}\right] N_{0}+n\left(\frac{N_{0}}{2}\right)^{2}\right] .
\end{gathered}
$$

The case of sending a symbol of "-1" may be computed in a likewise fashion; that is,

$$
\begin{gathered}
E\left[y_{l} \mid H, d_{l}=+1\right]=-E\left[y_{l} \mid H, d_{l}=-1\right], \\
\operatorname{Var}\left[y_{l} \mid H, d_{l}=+1\right]=\operatorname{Var}\left[y_{l} \mid H, d_{l}=-1\right] .
\end{gathered}
$$

Using (15) and assuming that follows a normal distribution under the given conditions, based on Gaussian approximation method [13], the conditional BER may be computed as

$$
\begin{aligned}
\operatorname{BER}\left[y_{l} \mid H\right]= & \frac{1}{4} \operatorname{erfc}\left(\frac{E\left[y_{l} \mid H, d_{l}=+1\right]}{\operatorname{Var}\left[y_{l} \mid H, d_{l}=+1\right]}\right) \\
& +\frac{1}{4} \operatorname{erfc}\left(\frac{-E\left[y_{l} \mid H, d_{l}=-1\right]}{\operatorname{Var}\left[y_{l} \mid H, d_{l}=-1\right]}\right),
\end{aligned}
$$

where $\operatorname{erfc}(\cdot)$ is the complementary error function.

The chebyshev map is used to generate chaotic sequence $x_{k}$ and we have $E\left[x_{k}^{2}\right]=0.5$ and $\operatorname{Var}\left[x_{k}^{2}\right]=0.125$. If $E_{b}$ is the bit energy and is represented by

$$
E_{b}=4 \beta E\left[x_{k}^{2}\right],
$$

for large $2 \beta$, the conditional BER may be simplified as

$$
\operatorname{BER}\left[y_{l} \mid \gamma_{b}\right] \approx \frac{1}{2} \operatorname{erfc}\left(\sqrt{\gamma_{b}\left(\frac{7}{2 \beta} \gamma_{b}+8+\frac{4 n \beta}{\gamma_{b}}\right)^{-1}}\right)
$$

where

$$
\gamma_{b}=\left(\frac{E_{b}}{N_{0}}\right) \sum_{i=1}^{n} h_{i}^{2} .
$$

Because $h_{i}$ is the Rayleigh distributed random variable, the probability density function (pdf) of $\gamma_{b}$ can be obtained as

$$
f\left(\gamma_{b}\right)=f\left(\gamma_{1}\right) \otimes f\left(\gamma_{2}\right) \otimes \cdots \otimes f\left(\gamma_{n}\right),
$$

where

$$
\begin{gathered}
\gamma_{i}=\left(\frac{E_{b}}{N_{0}}\right) h_{i}^{2}, \\
f\left(\gamma_{i}\right)=\bar{\gamma}^{-1} \exp \left(\frac{-\gamma_{i}}{\overline{\gamma_{i}}}\right), \\
\overline{\gamma_{i}}=\left(\frac{E_{b}}{N_{0}}\right) E\left[h_{i}^{2}\right],
\end{gathered}
$$

where $E\left[h_{i}^{2}\right]$ is the average power gain for the $i$ th channel. We assume that all independent channels satisfy the same Rayleigh fading channel condition; the pdf of $\gamma_{b}$ can be calculated as

$$
f\left(\gamma_{b}\right)=\left(\frac{\gamma_{b}^{n-1}}{\left(\bar{\gamma}_{i}^{n}(n-1) !\right)}\right) \exp \left(\frac{-\gamma_{b}}{\overline{\gamma_{i}}}\right),
$$

where $n$ is the number of receiver antennas. Finally, the BER of SIMO-CDSK system can be obtained by averaging the conditional BER; that is,

$$
\operatorname{BER}=\int_{0}^{+\infty} f\left(\gamma_{b}\right) \operatorname{BER}\left[y_{l} \mid \gamma_{b}\right] d \gamma_{b} .
$$

This formula will be used in the next section for evaluating the bit error rate performance of the proposed system.

\section{Simulation Results}

In this section, some numerical calculation, approximation, and simulation results of BER are presented at various conditions. To compare them with those analytical results in Section 3, Chebyshev map with degree of $\mu=3$ is adopted here. The parameters used in SIMO-CDSK system are the same as those in the conventional SISO-CDSK system and GCDSK system where the distinct chaotic sequences are generated by $x_{k+1}=4 x_{k}^{3}-3 x_{k}$ with initial value of $x_{0}=0.1$ and the average power gain of each Rayleigh fading channel $E\left[h_{i}^{2}\right]=1$. In order to facilitate the illustration, in the following figures, the conventional SISO-CDSK system is expressed by $\operatorname{CDSK}(1,1)$, the SIMO system is expressed by 


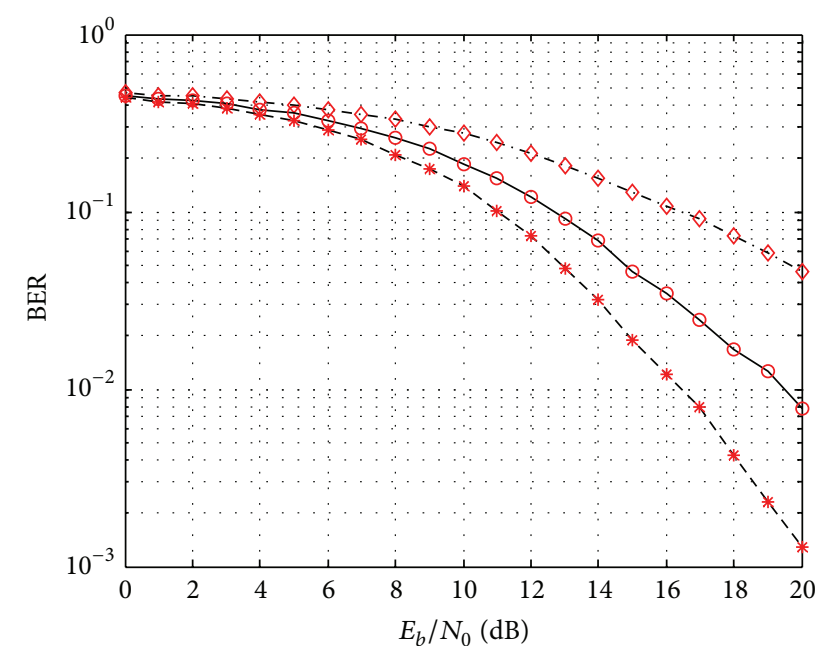

$\begin{array}{ll}-\cdots \operatorname{CDSK}(1,1) \text {, simulation } & \circ \operatorname{CDSK}(1,2) \text {, theory } \\ \diamond & \operatorname{CDSK}(1,1) \text {, theory } \\ -\operatorname{CDSK}(1,2) \text {, simulation } & *-\operatorname{CDSK}(1,3) \text {, simulation } \\ & * \operatorname{CDSK}(1,3) \text {, theory }\end{array}$

FIGURE 3: Relationships between BER performance and SNR for SISO-CDSK system and SIMO-CDSK system with the spreading factor $2 \beta=100$ and the time delay $L=200$, respectively.

$\operatorname{CDSK}(1, n), n$ means the number of receiver antennas, and the GCDSK system is expressed by GCDSK.

Figure 3 plots relationships between BER performance and SNR for SISO-CDSK system and SIMO-CDSK system with the spreading factor $2 \beta=100$ and the time delay $L=$ 200 , respectively. Theory and simulation results in this figure show that the analytical estimates which perform in Section 3 agree well with the simulation results; with the increase in the number of receiver antennas, at the fixed $E_{b} / N_{0}$ value, the proposed system could obtain more diversity gain; this is because, in $(18), \operatorname{erfc}(\cdot)$ is the decreasing function and the exception of correlator output grows faster than the square of double variance if the number of receiver antennas increases; when the $E_{b} / N_{0}$ value increases, further performance gain can also be expected by the proposed system.

Figure 4 plots the relationships between BER performance and the spreading factor $2 \beta$ for SISO-CDSK system and SIMO-CDSK system with $L=2 \beta$, and $E_{b} / N_{0}=10 \mathrm{~dB}$ and $15 \mathrm{~dB}$, respectively. Simulation results in this figure show that, for different $E_{b} / N_{0}$ values, the best performance of all systems can be obtained by selecting the critical point of spreading factors; for example, for $E_{b} / N_{0}=10 \mathrm{~dB}$, the critical point is nearly 20 ; for $E_{b} / N_{0}=15 \mathrm{~dB}$, the critical point is nearly 40 ; if the spreading factor is greater than the critical point, at the fixed $E_{b} / N_{0}$ value, BER performance for all systems becomes worse, which can be attributed to the increasing negative contribution from interference components in the correlator output which are shown in (10).

Figure 5 plots the relationships between BER performance and the time delay $L$ for SISO-CDSK system and SIMO-CDSK system with the spreading factor $2 \beta=100$ and $E_{b} / N_{0}=10 \mathrm{~dB}$ and $15 \mathrm{~dB}$, respectively. Simulation results in this figure show that, at the fixed $E_{b} / N_{0}$ value and $2 \beta$, the

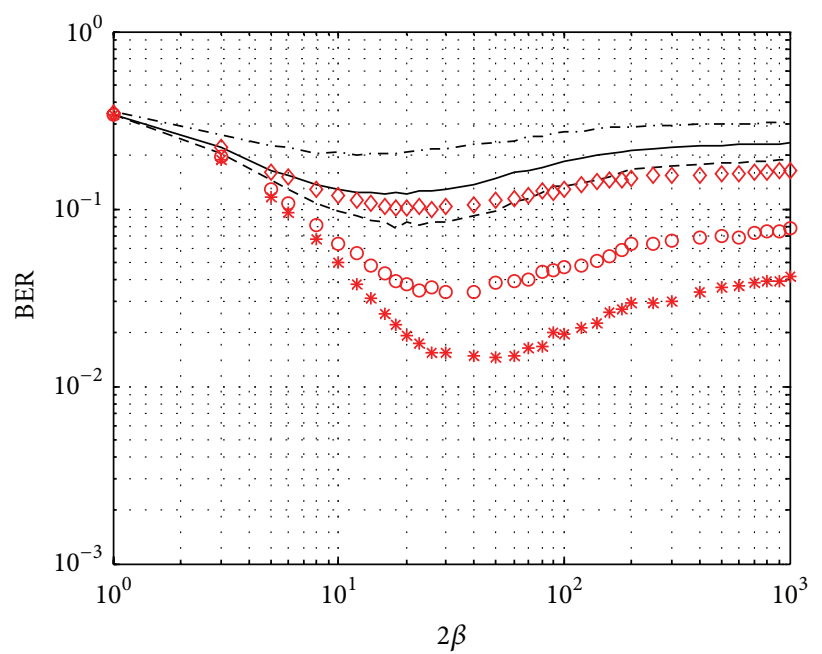

$\cdots \operatorname{CDSK}(1,1), E_{b} / N_{0}=10 \mathrm{~dB} \diamond \operatorname{CDSK}(1,1), E_{b} / N_{0}=15 \mathrm{~dB}$

$-\operatorname{CDSK}(1,2), E_{b} / N_{0}=10 \mathrm{~dB} \circ \operatorname{CDSK}(1,2), E_{b} / N_{0}=15 \mathrm{~dB}$

- - $\operatorname{CDSK}(1,3), E_{b} / N_{0}=10 \mathrm{~dB} * \operatorname{CDSK}(1,3), E_{b} / N_{0}=15 \mathrm{~dB}$

FIGURE 4: Relationships between BER performance and the spreading factor $2 \beta$ for SISO-CDSK system and SIMO-CDSK system with $L=2 \beta$ and $E_{b} / N_{0}=10 \mathrm{~dB}$ and $15 \mathrm{~dB}$, respectively.

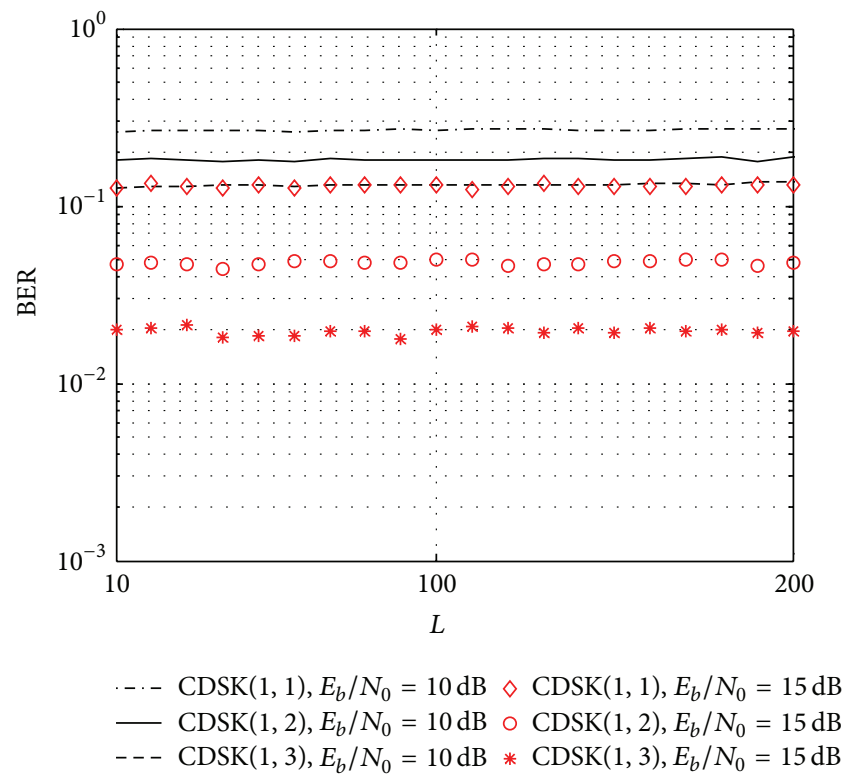

FIGURE 5: Relationships between BER performance and the time delay $L$ for SISO-CDSK system and SIMO-CDSK system with the spreading factor $2 \beta=100$ and $E_{b} / N_{0}=10 \mathrm{~dB}$ and $15 \mathrm{~dB}$, respectively.

diversity gain obtained by SIMO-CDSK system will not be affected by $L$. This is because the bit error rate (BER) formula, shown in (23), is independent of $L$. Therefore, we could choose a random number as the time delay for increasing the security of transmission.

Figure 6 plots the relationships between BER performance and SNR for SISO-CDSK system, SIMO-CDSK system, and GCDSK system. We assume that there are 


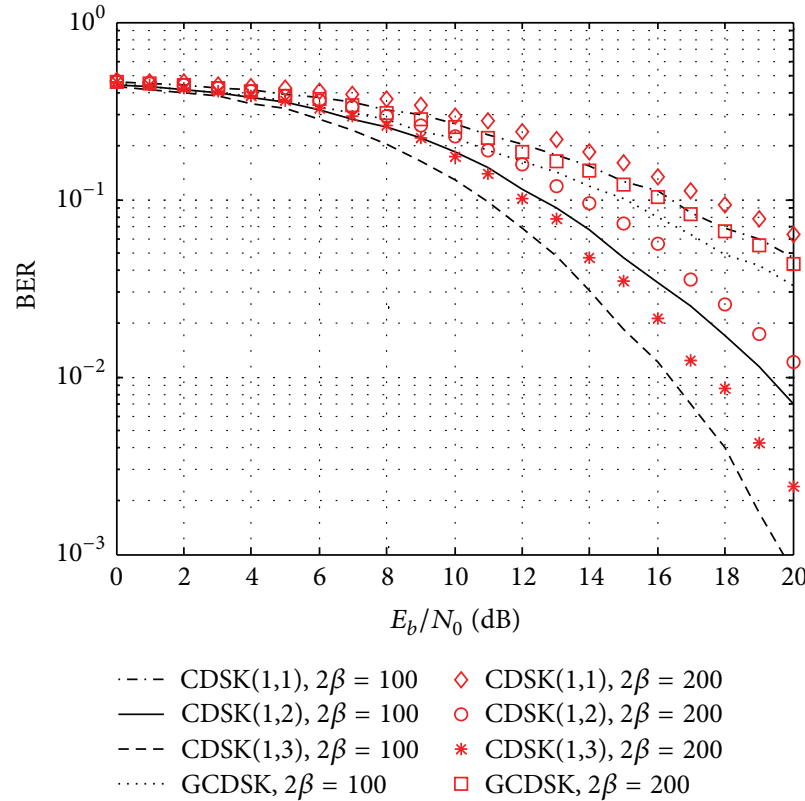

(a)

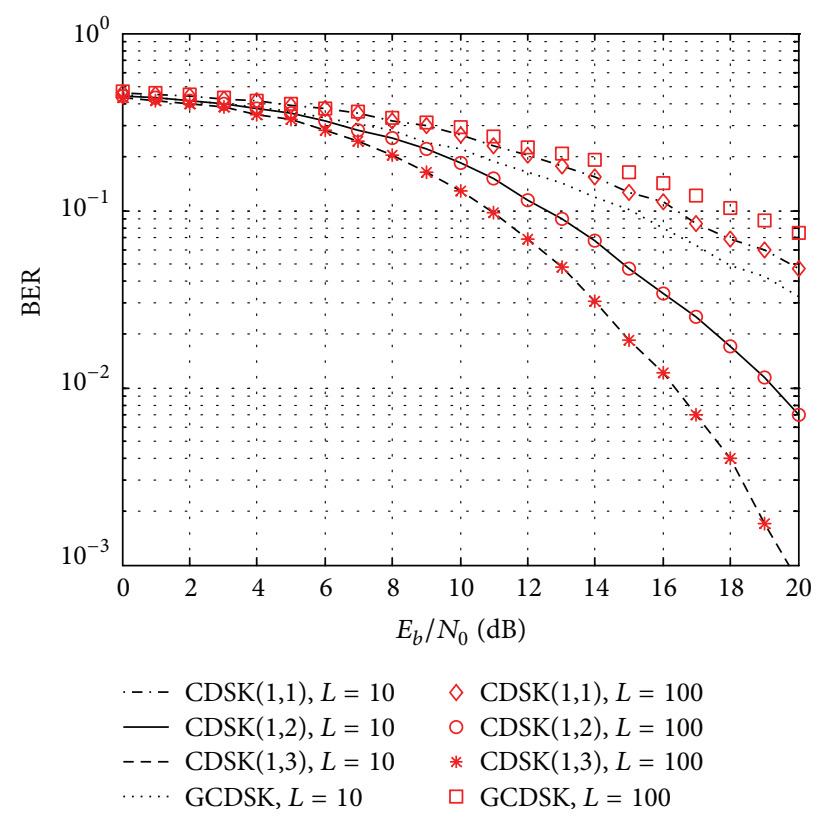

(b)

FIGURE 6: Relationships between BER performance and SNR for SISO-CDSK system, SIMO-CDSK system, and GCDSK system.

3 delay blocks in the transmitter of GCDSK. In Figure 6(a), the spreading factor $2 \beta$ considered here are 100 and 200 , respectively, and the time delay $L$ is set to 10 . Simulation results in this figure show that, at the fixed $E_{b} / N_{0}$ value, for different spreading factors, the BER performance of the proposed system is always superior to that of SISO-CDSK system and GCDSK system. In Figure 6(b), the spreading factor $2 \beta$ considered here is 100 and the time delay $L$ is set to 10 and 100 , respectively. In this figure, we can observe that, at the fixed $E_{b} / N_{0}$ value, for different time delays, the proposed system has an outstanding bit error rate performance in contrast to the conventional SISO-CDSK and GCDSK communication system. Moreover, from the simulated curve, it is also very interesting to observe that, since the intrasignal interference increases with the time delay, the BER performance of GCDSK becomes worse when $L$ increases, while the performance of SISO-CDSK system and the proposed system is not affected by $L$. Therefore, the proposed system has better security than that of GCDSK system.

\section{Conclusion}

In this paper, a single-input multiple-output (SIMO) architecture of correlation delay shift keying (CDSK) modulation technique is proposed as an enhanced version of CDSK, and its BER formula is also derived under the assumption of the proposed scheme over Rayleigh fading channels.

In the proposed scheme, multiple receiver antennas provide several independent copies of signals from the transmitter, and the equal-gain combiner (EGC) is adopted at the receiver to estimate the information symbol.
Theoretical analysis and simulations show that, at a higher signal-to-noise ratio (SNR), the proposed SIMO-CDSK architecture has an outstanding bit error rate performance in contrast to the conventional SISO-CDSK and GCDSK communication system; for the given SNR, the diversity gain of the proposed system will be improved with the number of receiver antennas increasing; for different SNRs, the best performance of the proposed system can be obtained by selecting the reasonable spreading factor; because the performance of SIMO-CDSK system is independent of the time delay, the proposed system has better security than that of GCDSK system.

\section{Acknowledgments}

This work was supported in part by the Natural Science Foundation of China under Grant 60874091, by the Specialized Research Fund for the Doctoral Program of Higher Education of China under Grant 20103223110003, and by the Six Projects Sponsoring Talent Summits of Jiangsu Province under Grant SJ209006.

\section{References}

[1] M. J. Ogorzalek, “Taming chaos. I. Synchronization,” IEEE Transactions on Circuits and Systems I, vol. 40, no. 10, pp. 693699, 1993.

[2] G. Kolumbán, B. Vizvki, W. Schwarz, and A. Abel, "Differential chaos shift keying: a robust coding for chaotic communication," in Proceedings of the Nonlinear Dynamics of Electronic Systems (NDES '96), pp. 87-92, Seville, Spain, 1996. 
[3] H. Yang and G. P. Jiang, "Reference-modulated DCSK: a novel chaotic communication scheme," IEEE Transactions on Circuits and Systems II, vol. 60, no. 4, pp. 232-236, 2013.

[4] M. Sushchik, L. S. Tsimring, and A. R. Volkovskii, "Performance analysis of correlation-based communication schemes utilizing chaos," IEEE Transactions on Circuits and Systems I, vol. 47, no. 12, pp. 1684-1691, 2000.

[5] W. N. Tam, F. C. M. Lau, and C. K. Tse, "Generalized correlationdelay-shift-keying scheme for noncoherent chaos-based communication systems," IEEE Transactions on Circuits and Systems I, vol. 53, no. 3, pp. 712-721, 2006.

[6] Q. Ding and J. N. Wang, "Performance analysis of correlationbased communication schemes utilizing chaos," IET Communication, vol. 5, no. 7, pp. 901-905, 2010.

[7] C. H. Jin and H. G. Ryu, "Performance evaluation of chaotic CDSK modulation system with different chaotic maps," in Proceedings of the International Conference on ICT Convergence, (ICTC '12), pp. 603-606, IEEE Press, Jeju Island, Republic of Korea, October 2012.

[8] Y.Xiao, MIMO: Multiple Antenna Communication System, Posts \& Telecom Press, Beijing, China, 1st edition, 2009.

[9] G. Kaddoum, M. Vu, and F. Gagnon, "Performance analysis of differential chaotic shift keying communications in MIMO systems," in Proceedings of the International Symposium of Circuits and Systems, (ISCAS '11), pp. 1580-1583, IEEE Press, Rio de Janeiro, Brazil, May 2011.

[10] Y. Fang, J. Xu, L. Wang, and G. Chen, "Performance of MIMO relay DCSK-CD systems over Nakagami fading channels," IEEE Transactions on Circuits and Systems I, vol. 60, no. 3, pp. 757-767, 2013.

[11] L. Wang, X. Min, and G. Chen, "Performance of SIMO FMDCSK UWB system based on chaotic pulse cluster signals," IEEE Transactions on Circuits and Systems I, vol. 58, no. 9, pp. 2259-2268, 2011.

[12] T. Geisel and V. Fairén, "Statistical properties of chaos in Chebyshev maps," Physics Letters A, vol. 105, no. 6, pp. 263-266, 1984.

[13] W. M. Tam, F. C. M. Lau, and C. K. Tse, Digital Chaotic Communication: Multi-Access Way and Performance Evaluation, Science Press, Beijing, China, 1st edition, 2007. 


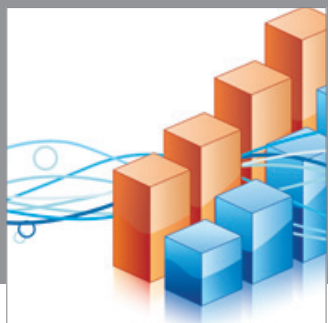

Advances in

Operations Research

mansans

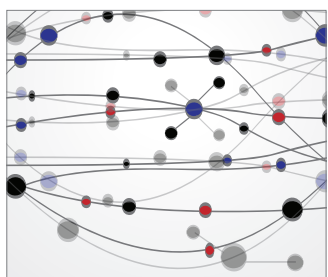

The Scientific World Journal
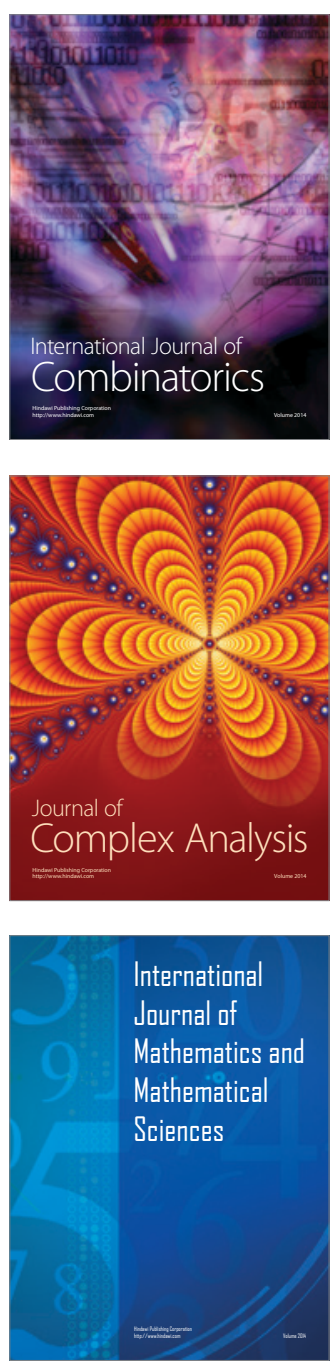
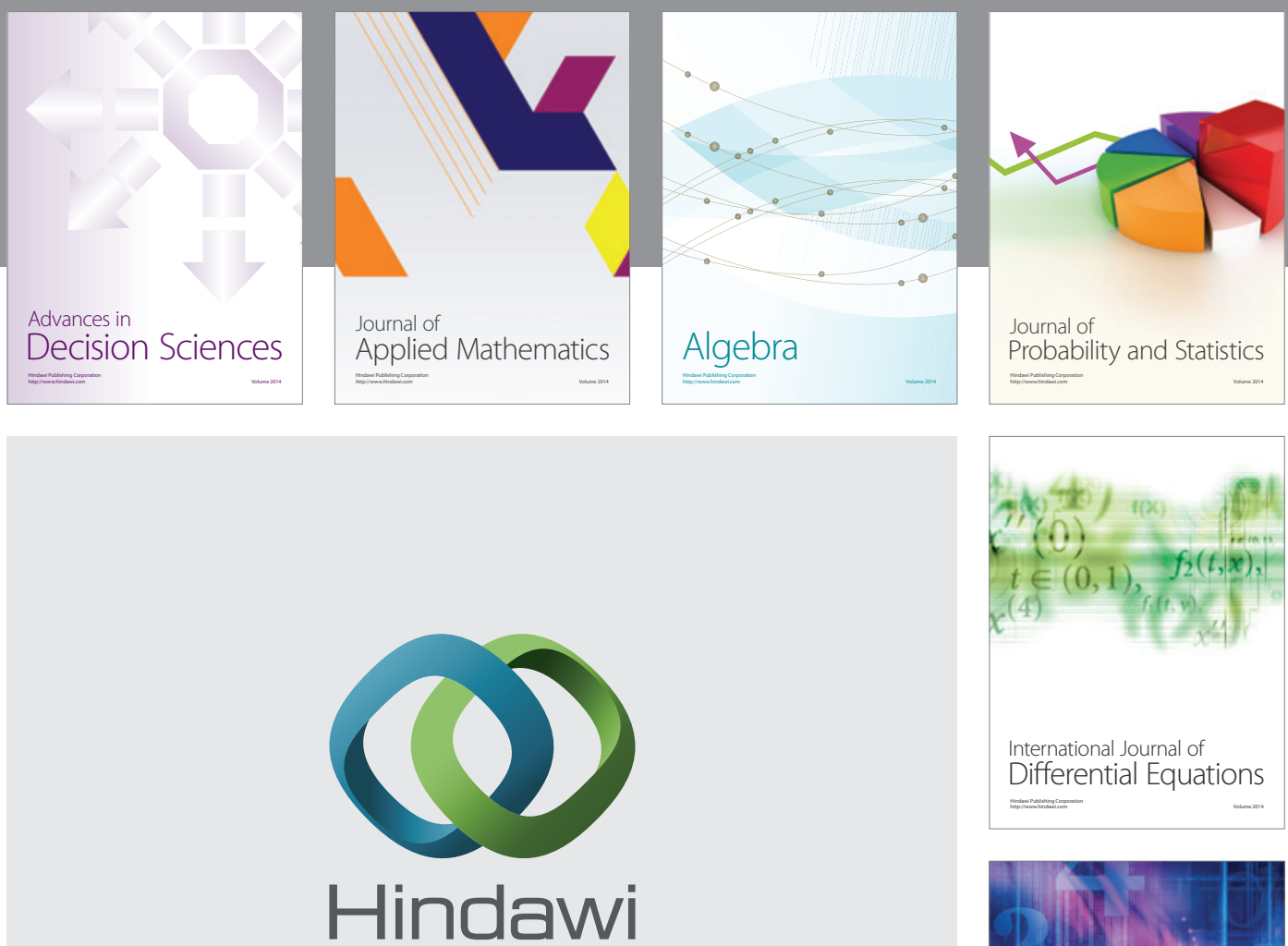

Submit your manuscripts at http://www.hindawi.com
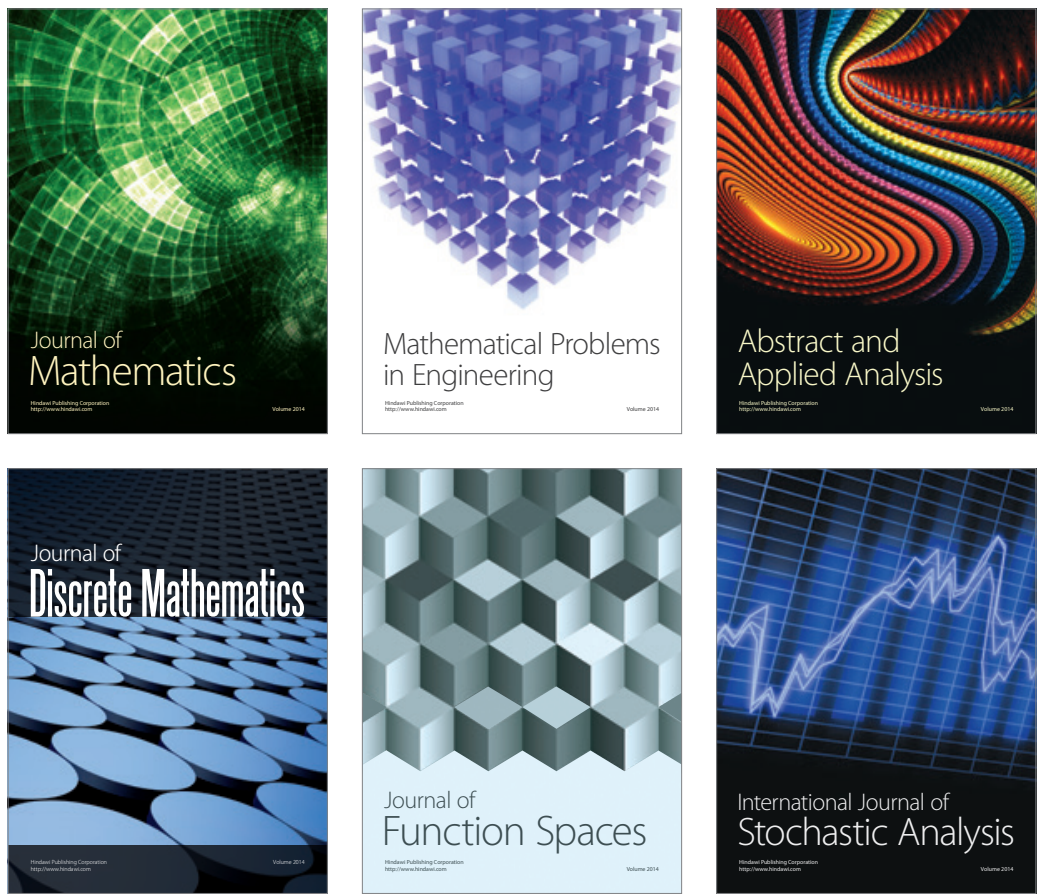

Journal of

Function Spaces

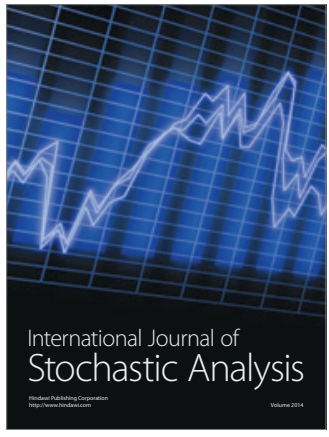

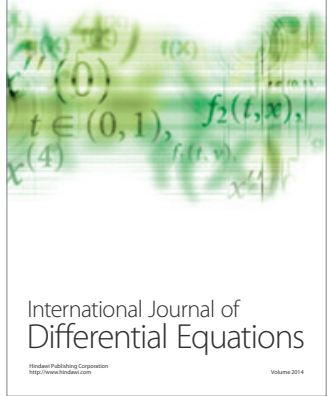
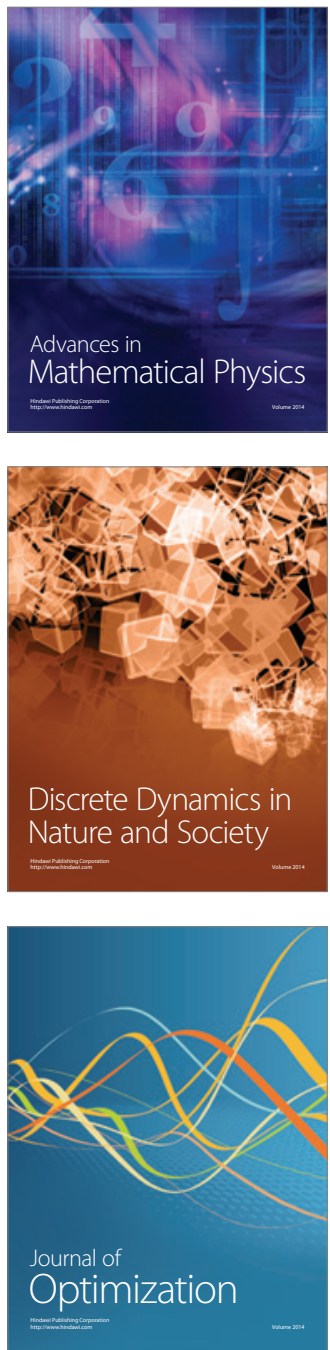